\title{
Outcomes Associated with Psychopathic Traits in a Non- Clinical Sample of Children Aged 6-13
}

\author{
Tracy G Cassels*, Susan AJ Birch \\ Department of Psychology, University of British Columbia, 2136 West Mall, Vancouver, BC V6T 1Z4, Canada
}

Received: November 22, 2013; Accepted: January 30, 2014; Published: January 31, 2014

*Corresponding author: Tracy G Cassels, Department of Psychology, University of British Columbia, 2136 West Mall, Vancouver, BC V6T 1Z4, Canada, E-mail: tracy@psych.ubc.ca

\begin{abstract}
Childhood psychopathy is a construct that remains contentious for many researchers, as questions still arise about the merit of assessing these traits in younger individuals, particularly in nonclinical children. Much of the research on psychopathy in children has focused on clinical samples, whereas few studies have examined the outcomes associated with various levels of these so-called 'psychopathic' traits in young, non-clinical children. In the current study, we assessed 129 non-clinical, elementary-school-aged children on perspective-taking, social-emotional well-being, and psychopathic traits. No relationships were found between psychopathic traits and perspective-taking, yet individual differences in the components of psychopathy were associated with multiple aspects of socialemotional well-being, though these changed across development. Considered within the broader literature on psychopathic traits in childhood and adolescence, the results suggest that the construct of psychopathy and its relationships to various outcomes or abilities varies across development.
\end{abstract}

Keywords: Psychopathy; Childhood; Perspective-taking; Socialemotional well-being; Community sample; Social outcomes; Prosocial behavior; Peer relationship problems; Emotion recognition

\section{Introduction}

Psychopathy has gained interest as a research topic in recent years due to the devastating effects it has on society and the frequent failure of traditional rehabilitation techniques. Psychopaths commit more crimes and a wider variety of crimes than other criminals, thereby placing a disproportionately large burden on society. Although only $20 \%$ of criminals are diagnosed with psychopathy, they commit approximately 50\% of all serious crimes [1]. Psychopathy is reportedly comprised of callous-unemotional traits (e.g., low empathy), impulsive/ antisocial behavior, and interpersonal features (e.g., narcissism) $[2,3]$. However, the defining aspects of psychopathy are argued to be the presence of callous-unemotional traits and interpersonal features, with some individuals showing impulsive/antisocial behaviour while others are able to use controlled behaviour to achieve their ends [4]. One of the major features of psychopathy is a deficit in socialization [5] such that individuals with these traits (e.g., egotism, reduced guilt) tend to have greater interpersonal problems and difficulties processing specific types of emotional situations or stimuli.

Previous research suggests that some children show personality traits and behaviors similar to adults identified as psychopaths, extending the concept of psychopathy downwards and using an adult definition when examining younger children [6-7]. There are also reasons to believe that the factors associated with psychopathy are relatively stable across development. For example, the callous-unemotional traits of psychopathy are potentially noticeable as early as age three [8] and may have a genetic basis [9]. Research into the stability of callousunemotional traits has found that levels of callous-unemotional traits are generally quite stable across childhood (examined as early as seven years of age) into adulthood [10-15], particularly for individuals with other concurrent diagnoses such as Conduct Disorder (e.g., [16]). Recently, Fontaine and colleagues identified four different trajectories for the presence of callous-unemotional traits from middle childhood to pre-adolescence [17]. Using a large twin dataset, the authors were able to determine that all four possible trajectories for these traits were found: stable-high, increasing, decreasing, or stable-low. The most common trajectory was stable-low ( $74.6 \%$ of the sample; $59.5 \%$ of which were girls) followed by decreasing (13.4\%; $37.4 \%$ girls) then increasing (7.3\%; $33.7 \%$ girls) and finally stable-high (4.7\%; $19.5 \%$ girls). Although genetics accounted for group membership in most cases, environment played a larger role for some (particularly the stable-high females). The presence of both stable and changing trajectories and the mixed influence of genetics and environment highlights the need to better understand the role of these traits, and their outcomes, at an early age and in non-clinical samples (as some of the non-clinical children may reach clinical status with increasing levels of psychopathic traits).

The results from [17] also suggest that 'psychopathy' or the presence of 'psychopathic traits' in childhood can be characterized as both dimensional and discrete. The dimensional view of psychopathy presupposes that there are meaningful individual differences both within those who reach clinical status and those that do not. For example, individuals rated as psychopathic by meeting a cutoff score will still show variability in the traits or 
behaviors based on the degree to which they surpassed the cutoff score. Similarly, individuals who are deemed non-psychopathic may still show different levels of behaviors and outcomes based on the degree of psychopathic traits they demonstrate. The discrete view of psychopathy assumes the most important qualitative differences are those between individuals who reach a particular cutoff score versus those who do not. Looking at childhood psychopathy, it seems both views are plausible and there is evidence for each. The presence of stable-high and stablelow trajectories suggest the possibility of a distinct subtype of individual with unique outcomes, whereas the low-high and high-low groups suggest a more continuous view of psychopathic traits that may or may not have meaningful outcomes (e.g., in social well-being) associated with the changing levels.

Much of the research into the outcomes associated with psychopathic traits utilizes the assumptions of the discrete view. For example, findings pertaining to low fear or reduced physiological responses to negative stimuli were found by comparing groups of children with Conduct Problems who also had either high or low levels of callous-unemotional traits (e.g., [18]), treating the distinction as discrete for clinical groups. These findings are important given that specific personality traits associated with psychopathy, namely callous-unemotional traits,now serve as a specifier for Conduct Disorder in the DSM-V [19], where a discrete view is necessary. However, this discrete view fails to capture the potentially subtle outcomes associated with varying levels of psychopathy in non-clinical samples (namely the dimensional view of psychopathy).

Herein, we are interested in outcomes associated with a dimensional view of psychopathy for non-clinical samples. In individual differences research, this type of dimensional view presupposes that there are meaningful differences in outcomes based on varying levels of certain traits and behaviors. Research on the outcomes associated with psychopathic traits in nonclinical children is limited, though the research that exists does support the dimensional view of psychopathy in non-clinical child populations [2,20] (but see [21] for an alternate view). Some research has been done on the construct of psychopathy in younger children (e.g., [9]), however, the focus tended to be on genetic factors or the mere presence/absence of psychopathy in conjunction with antisocial behavior. Research is still needed to examine the social, cognitive, and emotional correlates in for nonclinical children with varying levels of so-called psychopathic traits.

The research on childhood psychopathy that has focused exclusively on children and youths with clinical problems $[6,22]$ provides a starting point for examining which outcomes may be found in non-clinical samples. It remains unclear if these outcomes are associated with psychopathic traits, the combination of psychopathy and the secondary behavioral problems, or are simply a product of reaching a particular threshold with respect to psychopathic traits. With this in mind, previous research with clinical samples begs the question whether the social and emotional deficits observed appear, although to a lesser degree, in non-clinical samples with varying levels of psychopathic traits. For example, children with behavioral difficulties and psychopathic traits show reduced emotional responsivity to negative stimuli [23], which has been linked to deficits in amygdala function and in turn, empathy [24]. Similarly, Blair and colleagues have consistently found deficits in affective perspective-taking - specifically the ability to accurately identify negative emotions (e.g., sadness, fear) - in those with behavioral problems and high levels of psychopathic traits (as assessed by the Antisocial Process Screening Device ('APSD') $[25,26])$, despite evidence that psychopaths show no deficits in cognitive perspective-taking [27].

In addition to these emotional correlates of psychopathy, one of the primary aspects of psychopathy in clinical samples is a failure to form quality relationships $[5,3]$ instead, these relationships tend to be superficial in nature. One way to assess these relationships is to examine social-emotional well-being. Social-emotional well-being includes both the interpersonal (e.g., expressing affection towards others) and intrapersonal (e.g., regulating one's own emotions and behaviors) components that affect our social relationships. Importantly, these aspects of wellbeing have been identified as protective factors; keeping adults who are at high-risk for psychopathy and antisocial behavior out of the criminal justice system [28], with higher levels of social relationships and social competence resulting in lower levels of criminal behavior.

Examining these specific effects of psychopathy in non-clinical child populations is warranted in an attempt to understand how varying levels of these traits affect the functioning of non-clinical groups of children. These findings are particularly important for research purposes where assumptions about the applicability of clinical outcomes to non-clinical samples may in fact be inappropriate. Importantly, when looking at perspective-taking and social-emotional well-being, there is research linking deficits in these abilities to psychopathic traits in non-clinical samples, but not in childhood. For example, [29] found relationships between psychopathic traits and affective perspective-taking deficits in a non-clinical pre-adolescent and adolescent sample, but there has not yet been any research to determine if these findings hold in younger children.

The current study aimed to examine outcomes associated with psychopathic traits (specifically perspective-taking and social-emotional well-being) in a younger-than-previously-tested group of non-clinical children. Utilizing the dimensional view of psychopathy, we predicted that variability of psychopathic traits in this non-clinical sample would be associated with changes in perspective-taking abilities and social-emotional well-being (i.e., higher levels of psychopathic traits would be associated with greater deficits). To this end, we assessed the traits associated with psychopathy, specifically callous-unemotional traits, narcissism, and conduct problems, in a group of children and youths (aged five to 13) and their relationships to both perspective-taking and social-emotional well-being. Given that the majority of work with children of this age has been done with those with concurrent behavioral problems, it is necessary to test the hypothesis that the relationships found in clinical samples (often with co-morbid 
conditions) also hold for the non-clinical samples tested here and whether they vary depending on the levels of psychopathic traits. We hypothesized that 1) children with greater psychopathic traits will show decreased perspective-taking and 2) children with greater psychopathic traits will show decreased socialemotional well-being, though we did not expect to see severe deficits in either perspective-taking nor social-emotional wellbeing given the non-clinical nature of the sample.

\section{Materials and Methods}

\section{Participants}

Participants were 129 elementary-school-aged students (70 male) living in the Greater Vancouver Area in Canada, ranging in age from 5 years 7 months to 13 years 3 months (M=8yr 11mos) and were predominantly Caucasian $(54.3 \%)$ or Asian $(27.9 \%)$; $6.2 \%$ declined to answer; the remainder were of various ethnicities. Twenty-three students were previously diagnosed with a learning disorder (LD) and thus LD status was examined to see if its presence affected the results. Not all participants and their parents completed all portions of the study, thus results are presented for the data available for each measure. Data were missing for the Strengths and Difficulties Questionnaire ( $n=60$ available), the Reading the Mind in the Eyes Task ( $n=121)$, the generative version of the Reading the Mind in the Eyes Task $(n=122)$, and the [30], Vocabulary Measures ( $n=120)$; full data were available for the Antisocial Process Screening Device.

\section{Parent-Report Measures}

Antisocial Process Screening Device (APSD; [31]): The APSD is a parent-report 20-item questionnaire scored on a threepoint scale (i.e., Never True, Sometimes True, Always True). It provides scores on three subscales assumed to represent the factors of psychopathy in children - Narcissism, CallousUnemotional (CU) Traits, and Impulsive/Antisocial Behavior and a full scale 'Psychopathy' factor, with good internal reliability in a community sample [2]. The APSD has been found to have predictive validity for children aged six to 13 and is one of the most commonly used screening devices for psychopathy in children and adolescents. In the current sample, the alpha for the entire measure was 0.695 (CU Traits: $\alpha=0.412$, Narcissism $\alpha=0.613$, Impulsivity $\alpha=0.583$ ).

Strengths and Difficulties Questionnaire (SDQ; [32,33]): This parent-report questionnaire consists of 25 items on a three-point scale (i.e., Not True, Somewhat True, Certainly True) and provides one Total Difficulties score and five different components of social-emotional well-being: Emotional Symptoms, Conduct Problems, Hyperactivity/Inattention, Peer Relationship Problems, and Prosocial Behavior. This measure assesses a range of child behavior problems and is appropriate for children aged two to 21 . This is a widely used measure of social-emotional well-being, and has excellent psychometric properties (e.g., [33]). In the current sample, internal reliability was as follows: Emotional Symptoms $\alpha=0.662$, Conduct Problems $\alpha=0.653$, Hyperactivity/Inattention $\alpha=0.742$, Peer Relationship Problems $\alpha=0.610$ and Prosocial Behavior $\alpha=0.708$.

\section{Child Tasks}

Reading the mind in the eyes - children's version [34] (henceforth the 'Eyes Task'): This task requires child participants to choose which mental or emotional state (from a selection of four terms) a person is displaying based on pictures of the eyeregion. For each picture children are asked, 'What is this person feeling?' The full version includes 28 pictures from the adult version of the task selected based on the performance of a sample of non-clinical children [34]. In the current study, half of the images were given in this forced-choice format and the other half were administered in an open-ended format (described next).

The generative eyes task - children's version [35,36] (henceforth the GET): In this open-ended modification of [34]'s Eyes Task the participant must spontaneously generate the answer to the question 'What is this person feeling?' without any prompting terms, creating a more ecologically valid and specific measure of emotional perspective-taking [35]. The inclusion of the GET allows for examination of children's perspective-taking abilities in the absence of compensatory strategies such as process of elimination that are possible with the forced-choice version of the task.

Woodcock-Johnson III vocabulary measures [30]: Participants were given these measures to control for verbal ability. The verbal ability score from the WJ III correlates $r=0.73$ with the verbal IQ score and $r=0.68$ with the full-scale IQ from the WISC-III [37].

\section{Procedure}

Consent and verbal assent was obtained by parents and children, respectively. Children first received the GET, then the Eyes Task, and finally the WJ-III. Parents completed their questionnaires either in the lab or at home. The study was conducted after approval by the University's Behavioral and Research Ethics Board.

\section{Results \\ Preliminary results}

There were no gender differences on any of the relevant scales or measures (all $p$ 's $>0.05$ ). There were no differences by LD status on any of the APSD subscales, but there was a significant difference by LD status on the Peer Relationship Problems subscale of the SDQ ( $p=0.022)$ even after controlling for age and verbal mental age, thus LD status was included for any further analysis involving the Peer Relationship Problems subscale. Furthermore, age was significantly related to verbal mental age $(r=0.570, p<0.001)$, the CU subscale of the APSD ( $r=0.211$, $p=0.019)$, and total correct for the Eyes Task $(r=0.250, p=0.006)$. Verbal mental age was related to a subset of the same variables as age, and given the moderate-large correlation between chronological age and verbal mental age, only chronological age is included as a covariate in future analyses. See Table 1 for all means and standard deviations.

\section{Psychopathic traits and perspective-taking}

We examined individual differences in psychopathic traits to 
see if they predicted individual differences in perspective-taking abilities, as assessed by the Eyes Task and the GET. Hierarchical linear regression analyses were performed with age included as the first step of the equation and then psychopathy (as measured by the APSD) in the second step. Psychopathy as a whole significantly predicted performance on the GET, $\Delta R^{2}=0.035$, $p=0.039$, but it was in an unexpected direction-children who scored higher on the measure of psychopathy showed better affective perspective-taking skills than children who performed worse, $\beta=0.188$. When the individual subscales of psychopathy were entered independently, only Narcissism was found to be a significant predictor of the GET, $\Delta R^{2}=0.037, p=0.043$, again in an unexpected direction-higher narcissism was related to a better performance on the GET. See Table 2 for full results.

To more fully examine these relationships using categories of psychopathic traits, scores on the APSD were categorized by those who scored below ('low') or above ('moderate') the norm for a community sample [2]. The scores on the perspective-taking measures were then compared between these two groups to see if differences existed between these two non-clinical groups. Independent samples t-tests were used to compare scores on the various perspective-taking measures. The only significant difference was for the GET when examined by Narcissism, again with the group that scored higher on Narcissism outperforming the lower group. See Table 3 for full results. This would suggest that regardless of the degree of psychopathic traits within a normative range, one's perspective-taking abilities are not affected in this age group, although there is the potential for greater Narcissism to be related to greater affective perspective-

Table 1: Descriptive Data of Measures.

\begin{tabular}{|c|c|c|c|c|}
\hline & Minimum & Maximum & Mean & SD \\
\hline Chronological Age (months) & 67 & 159 & 107.34 & 25.01 \\
\hline Verbal Mental Age (months) & 65 & 288 & 127.46 & 34.16 \\
\hline \multicolumn{5}{|l|}{ APSD } \\
\hline Total Psychopathy & 0 & 22 & 9.28 & 4.12 \\
\hline Callous-Unemotional Traits & 0 & 8 & 2.50 & 1.66 \\
\hline Narcissism & 0 & 8 & 2.88 & 2.13 \\
\hline Impulsivity & 0 & 10 & 3.90 & 1.86 \\
\hline \multicolumn{5}{|l|}{ SDQ } \\
\hline $\mathrm{CP}$ & 0 & 6 & 1.05 & 1.45 \\
\hline $\mathrm{HI}$ & 0 & 9 & 3.31 & 2.33 \\
\hline PRP & 0 & 6 & 1.28 & 1.53 \\
\hline ES & 0 & 8 & 1.69 & 1.75 \\
\hline $\mathrm{PB}$ & 4 & 10 & 8.37 & 1.71 \\
\hline \multicolumn{5}{|l|}{ Eyes Tasks } \\
\hline ET & 3 & 14 & 9.12 & 2.04 \\
\hline GET & 0 & 9 & 5.38 & 1.88 \\
\hline
\end{tabular}

Note: APSD = Antisocial Process Screening Device; SDQ = Strengths and Difficulties Questionnaire; $\mathrm{CP}=$ Conduct Problems; HI = Hyperactivity/ Impulsivity; PRP = Peer Relationship Problems; ES = Emotional Symptoms; $\mathrm{PB}=$ Prosocial Behaviour; GET = Generative Eyes Task; ET = Eyes Task
Table 2: Regression results between psychopathic traits and perspectivetaking.

\begin{tabular}{|c|c|c|c|c|}
\hline \multirow{2}{*}{} & \multicolumn{2}{|c|}{ Predicting the GET } & \multicolumn{2}{c|}{ Predicting the ET } \\
\cline { 2 - 5 } & $\boldsymbol{\Delta R}^{\boldsymbol{2}}$ & $\boldsymbol{p}$-value & $\boldsymbol{\Delta \boldsymbol { R } ^ { 2 }}$ & $\boldsymbol{p}$-value \\
\hline CU Traits & 0.006 & 0.388 & 0.006 & 0.372 \\
\hline Narcissism & 0.034 & 0.043 & 0.000 & 0.886 \\
\hline Impulsivity & 0.017 & 0.148 & 0.003 & 0.527 \\
\hline Total Psychopathy & 0.035 & 0.039 & 0.003 & 0.567 \\
\hline
\end{tabular}

Note: GET=Generative Eyes Task; ET=Eyes Task. In all cases, age was entered in the first step of the equation while the component of psychopathy was entered in the second step.

Table 3: Mean differences on perspective-taking by 'low' and 'moderate' APSD scores.

\begin{tabular}{|c|c|c|c|c|c|}
\hline & & $\mathbf{M}_{\text {low }}$ & $\mathbf{M}_{\text {moderate }}$ & t-value & $p$-value \\
\hline \multirow{2}{*}{$\begin{array}{c}\text { CU Traits } \\
\left(\mathrm{N}_{\text {low }}=66 ; \mathrm{N}_{\text {mod }}=55\right)\end{array}$} & GET & 5.41 & 5.34 & 0.203 & 0.839 \\
\hline & ET & 9.17 & 9.07 & 0.251 & 0.802 \\
\hline \multirow{2}{*}{$\begin{array}{c}\text { Narcissism } \\
\left(\mathrm{N}_{\text {low }}=76 ; \mathrm{N}_{\text {mod }}=46\right)\end{array}$} & GET & 5.07 & 5.89 & -2.393 & 0.018 \\
\hline & ET & 9.28 & 8.87 & 1.075 & 0.284 \\
\hline \multirow{2}{*}{$\begin{array}{c}\text { Impulsivity } \\
\left(\mathrm{N}_{\mathrm{low}}=78 ; \mathrm{N}_{\bmod }=44\right)\end{array}$} & GET & 5.23 & 5.64 & -1.144 & 0.255 \\
\hline & ET & 9.19 & 9.00 & 0.495 & 0.622 \\
\hline \multirow{2}{*}{$\begin{array}{l}\text { Total Psychopathy } \\
\left(\mathrm{N}_{\text {low }}=76 ; \mathrm{N}_{\text {mod }}=46\right)\end{array}$} & GET & 5.22 & 5.63 & -1.158 & 0.249 \\
\hline & ET & 9.30 & 8.82 & 1.255 & 0.212 \\
\hline
\end{tabular}

Note: GET = Generative Eyes Task; ET = Eyes Task

taking abilities. Indeed, there is research that children with high scores on Machiavellianism (another cluster of personality traits that includes being manipulative, lying, high sense of self, etc.) showed high levels of affective perspective-taking, despite presenting with low levels of empathy [38].

\section{Psychopathy and social-emotional well-being}

Regression analyses were conducted predicting the components of and the Total Difficulties score of the SDQ of age (step one) and either the individual components of the ASPD (i.e., Callous-Unemotional Traits, Narcissism, and Impulsivity) or the Total Psychopathy score from the ASPD (step two). See Table 4a for full results. With the exception of Peer Relationship Problems, both the components of psychopathy and the Total Psychopathy score strongly predicted the social and emotional outcomes assessed by the SDQ, accounting for between 10 and $40 \%$ of the variance in the SDQ components ${ }^{1}$. An examination of the beta coefficients revealed that different components of psychopathy contributed to different outcomes. Higher CU Traits were related to lower Prosocial Behavior and lower Emotional Symptoms scores; this is not surprising given that CU traits have been linked with a reduction in overt displays of emotions, notably negative emotions [2,3], and negative emotions are assessed herein by the Emotional Symptoms subscale. Narcissism was related to higher Conduct Problems and Total Difficulties scores as well as lower Prosocial Behavior scores, whereas Impulsivity was related to

1Not that the Peer Relationship Problem score may be affected by the LD group differences, although LD status was included as a covariate, so the likelihood is small. 
higher Conduct Problems, Hyperactivity/Inattention, Emotional Symptoms, and Total Difficulties scores. See Table $4 \mathrm{~b}$ for full results.

Although providing support for the hypothesis that psychopathic traits, even in non-clinical ranges, can have an effect on social functioning, the analyses thus far do not, unfortunately, tell us whether the higher scores on the APSD scales reflect changes in actual difficulties in social-emotional functioning. We found that individual differences in psychopathic traits did predict changes in social-emotional functioning, nonetheless this did not speak to the issue of whether or not the changes were 'practically meaningful'. That is, some changes may be statistically meaningful but fail to capture a change that would be noticeable in daily functioning. To address this, social-emotional functioning scores for participants who scored above or below the mean on the APSD scales were compared, using a Bonferroni correction with a family-wise alpha of .10 to control for the multiple comparisons. See Table 5 for full results. For analyses involving children who scored above or below the mean of the Total Psychopathy scale, there were significant differences in Hyperactivity/Inattention and Prosocial Behavior, but only the differences in Prosocial Behavior were representative of categorically different difficulty levels as defined by research on the SDQ [33], with those scoring above the mean on Total Psychopathy displaying moderate difficulties and those scoring below the mean displaying low difficulties. For CU Traits, there was a significant difference in Prosocial Behavior, but this difference did not predict a categorical change in difficulty level with both groups having low difficulties. For Narcissism, the Prosocial Behavior scale showed a near-significant difference and also represented a

Table 4a: Regression predicting psychopathic traits from socialemotional health.

\begin{tabular}{|c|c|c|c|c|}
\hline \multirow{2}{*}{} & \multicolumn{2}{|c|}{ All subscales* } & \multicolumn{2}{c|}{ Total Psychopathy } \\
\cline { 2 - 5 } & $\boldsymbol{\Delta R}^{\mathbf{2}}$ & $\boldsymbol{p}$-value & $\boldsymbol{\Delta R}^{\mathbf{2}}$ & $\boldsymbol{p}$-value \\
\hline Conduct Problems & 0.323 & $<0.001$ & 0.283 & $<0.001$ \\
\hline Hyperactivity/Impulsivity & 0.406 & $<0.001$ & 0.319 & $<0.001$ \\
\hline Peer Relationship Problems** & 0.065 & 0.300 & 0.008 & 0.502 \\
\hline Prosocial Behavior & 0.405 & $<0.001$ & 0.338 & $<0.001$ \\
\hline Emotional Symptoms & 0.274 & 0.001 & 0.096 & 0.021 \\
\hline Total Difficulties & 0.428 & $<0.001$ & 0.305 & $<0.001$ \\
\hline
\end{tabular}

Note: *All subscales include Callous-Unemotional Traits, Narcissism, and Impulsivity. ${ }^{* *}$ Includes LD group status as a covariate.

Table 4b: Beta coefficients in predicting psychopathic traits.

\begin{tabular}{|c|c|c|c|c|}
\hline & CU Traits & Nar. & Imp. & Psychopathy \\
\hline Conduct Problems & 0.095 & $\mathbf{0 . 3 0 8}$ & $\mathbf{0 . 3 1 8}$ & $\mathbf{0 . 5 3 3}$ \\
\hline Hyperactivity/Impulsivity & 0.069 & 0.151 & $\mathbf{0 . 5 2 9}$ & $\mathbf{0 . 5 6 6}$ \\
\hline $\begin{array}{c}\text { Peer Relationship } \\
\text { Problems }\end{array}$ & -0.105 & 0.271 & -0.072 & 0.090 \\
\hline Prosocial Behavior & $\mathbf{- 0 . 3 9 7}$ & $\mathbf{- 0 . 3 7 5}$ & -0.111 & $\mathbf{- 0 . 5 8 2}$ \\
\hline Emotional Symptoms & $\mathbf{- 0 . 2 5 3}$ & 0.142 & 0.435 & $\mathbf{0 . 3 1 1}$ \\
\hline Total Difficulties & -0.062 & $\mathbf{0 . 2 9 2}$ & 0.469 & $\mathbf{0 . 5 5 3}$ \\
\hline
\end{tabular}

Note: Significant beta coefficients are in bold.
Table 5: Mean differences on social-emotional health by 'low' and 'moderate' APSD scores.

\begin{tabular}{|c|c|c|c|c|c|}
\hline & & $\mathbf{M}_{\text {low }}$ & $\mathbf{M}_{\text {moderate }}$ & t-value & $p$-value \\
\hline \multirow{6}{*}{$\begin{array}{c}\text { CU Traits } \\
\left(\mathrm{N}_{\text {low }}=27 ; \mathrm{N}_{\bmod }=33\right)\end{array}$} & Total Difficulties & 7.33 & 7.32 & -0.008 & 0.994 \\
\hline & ES & 2.04 & 1.40 & -1.412 & 0.163 \\
\hline & $\mathrm{CP}$ & 0.81 & 1.24 & 1.136 & 0.261 \\
\hline & $\mathrm{HI}$ & 3.07 & 3.50 & 0.698 & 0.488 \\
\hline & PRP & 1.41 & 1.18 & -0.565 & 0.574 \\
\hline & PB & 9.07 & 7.79 & -3.109 & 0.003 \\
\hline \multirow{6}{*}{$\begin{array}{c}\text { Narcissism } \\
\left(\mathrm{N}_{\text {low }}=41 ; \mathrm{N}_{\mathrm{mod}}=19\right)\end{array}$} & Total Difficulties & 5.93 & 10.34 & 2.737 & 0.012 \\
\hline & ES & 1.37 & 2.37 & 1.772 & 0.089 \\
\hline & $\mathrm{CP}$ & 0.73 & 1.74 & 2.058 & 0.052 \\
\hline & $\mathrm{HI}$ & 2.76 & 4.50 & 2.526 & 0.018 \\
\hline & PRP & 1.07 & 1.74 & 1.582 & 0.119 \\
\hline & $\mathrm{PB}$ & 8.78 & 7.47 & -2.930 & 0.005 \\
\hline \multirow{6}{*}{$\begin{array}{c}\text { Impulsivity } \\
\left(\mathrm{N}_{\text {low }}=41 ; \mathrm{N}_{\mathrm{mod}}=19\right)\end{array}$} & Total Difficulties & 5.77 & 10.68 & 3.363 & 0.002 \\
\hline & ES & 1.27 & 2.14 & 2.432 & 0.022 \\
\hline & $\mathrm{CP}$ & 0.73 & 1.74 & 2.189 & 0.038 \\
\hline & HI & 2.60 & 4.84 & 3.867 & $<0.001$ \\
\hline & PRP & 1.17 & 1.53 & 0.835 & 0.407 \\
\hline & PB & 8.66 & 7.74 & -1.994 & 0.051 \\
\hline \multirow{6}{*}{$\begin{array}{l}\text { Total Psychopathy } \\
\left(\mathrm{N}_{\text {low }}=40 ; \mathrm{N}_{\text {mod }}=20\right)\end{array}$} & Total Difficulties & 6.07 & 9.85 & 2.494 & 0.019 \\
\hline & ES & 1.51 & 2.05 & 0.964 & 0.344 \\
\hline & $\mathrm{CP}$ & 0.83 & 1.50 & 1.489 & 0.148 \\
\hline & $\mathrm{HI}$ & 2.56 & 4.80 & 3.917 & $<0.001$ \\
\hline & PRP & 1.17 & 1.50 & 0.773 & 0.443 \\
\hline & PB & 9.00 & 7.10 & -4.749 & $<0.001$ \\
\hline
\end{tabular}

Note: When appropriate, Case 4 t-tests were conducted when homogeneity of variance was not met. With a Bonferroni correction, any p-value at or less than 0.004 remains significant.

ES=Emotional Symptoms; $\mathrm{CP}=$ Conduct Problems; $\mathrm{HI}=$ Hyperactivity/ Inattention; PRP=Peer Relationship Problems; $\mathrm{PB}=$ Prosocial Behaviour

change in difficulty category, from low to moderate. Although the differences in Total Difficulties between those who scored above or below the mean on Narcissism were not significant after the Bonferroni correction, the moderate group scored at the very upper limit of low difficulties whereas the low difficulty group scored in the middle. Finally, for Impulsivity there were differences in Total Difficulties and Hyperactivity/Inattention. Both groups, however, scored as having low difficulties for both categories, but similar to what was found in Total Difficulties for Narcissism, the moderate group scored at the very upper limit of low difficulties whereas the low difficulty group scored in the middle.

\section{Discussion}

The primary goal of the current study was to examine the outcomes associated with the construct of psychopathy in a non-clinical sample of young children. Much of the work on this construct has focused exclusively on older children or 
adolescents, and individuals who have come into contact with the legal system or been labeled as having significant behavioral problems. Although there is good reason to research these clinical samples, given their more pronounced effects on society, knowledge that the trajectory for psychopathic traits can change across development [17] warrants research into non-clinical samples. As the trajectory of psychopathic traits for a given child can change from low to high and vice versa, understanding the outcomes for non-clinical samples may be one way to help identify those who are more or less likely to follow the lowto-high trajectory. There have been previous studies utilizing community samples, but none have examined the specific outcomes of interest herein-namely perspective-taking and social-emotional competence with the age-range utilized herein.

Our results were mixed with respect to the relationships between psychopathic traits and perspective-taking, thus it may be premature to think of all younger children with these traits as sharing the negative outcomes associated with these traits in older individuals. Results with respect to social-emotional well-being were more in line with those found in older samples [5]. Specifically, individual differences in social-emotional wellbeing were related to both the individual components and total score for psychopathy. Children who were rated as having higher psychopathic traits also showed greater problems with prosocial behavior and, to a lesser degree, emotional symptoms and conduct problems. These data are consistent with the notion that the reduction of prosocial behavior, long believed to be strongly related to empathy [39], is the key to identifying younger children at-risk for developing more serious antisocial behaviors and disorders later in development. Psychopathic traits were also related to the total reported number of social and emotional difficulties, suggesting that children with higher psychopathic traits exhibit greater difficulties in several socialemotional aspects of life. These findings highlight that even in non-clinical samples the presence of these traits impacts the child's interpersonal and intrapersonal well-being.

Notably, we did not find a linear relationship with respect to narcissism and social-emotional well-being. Rather, moderate levels of narcissism were associated with the least socialemotional deficits whereas the lowest levels were related to moderate or normal social-emotional functioning. In comparison, high levels of narcissism were linked with high levels of psychopathology. Although it is often thought that narcissism is a negative trait, these results could suggest that humans require some degree of narcissism to help build healthy relationships and function in a social world. One possibility for this is that higher levels of these traits provide a protection to the individual that allows them to rebound from negative social events instead of internalizing them.

In contrast, with our younger sample we did not reproduce previous findings [29] with older participants linking affective perspective-taking deficits with levels of psychopathic traits. This could be a result of the different methods used to assess affective perspective-taking (i.e., [29] used a computerized, forced-choice task with whole faces whereas the tasks in our procedure utilized only the eye-region of faces). However, given the previously reported strength of this deficit in psychopathy, one would expect to see this result emerge across various methods. A second possibility for the failure to reproduce the findings is that our sample did not include many individuals who were high on both callous-unemotional traits and impulsivity/aggression. This possibility is in line with other results [40] which found that deficits in affective perspective-taking were limited only to those children high on aggression and callous-unemotional traits.

Similarly, the lack of any highly aggressive/impulsive children in the sample could also suggest that any higher levels of the core elements of psychopathy (callous-unemotional traits and interpersonal features) may be associated with the more "successful" side of psychopathic traits [4]. Without the associated impulsivity or problematic behaviours, higher levels of the interpersonal features and callous-unemotional traits can be found in some individuals with successful careers (e.g., $[41,42])$. Affective perspective-taking, though often linked with empathy, is not causally related and therefore it is possible that for individuals high on the interpersonal features and callousunemotional traits of psychopathy, the ability to identify an emotion is less related to the ability to feel for the other individual (for supporting research with Machiavellianism, see [38]).

A final possibility is that the younger age of our sample contributed to the lack of finding affective perspective-taking deficits and that this particular deficit only becomes apparent in older children. Given that the identification of emotions continues to develop into adolescence [43], it is possible that the deficits associated with psychopathic traits mirror this protracted development. Extensions of psychopathy to young children using a dimensional view must consider that some of the core deficits and associations of the construct in older adolescents and adults may not be applicable to these younger individuals. This would not be the first time that a downward extension of outcomes was not replicated in a younger sample. For example, [44] examined parent-reported psychopathic traits and empathy in a community sample of 3- to 13-year old children. Contrary to results with adults and youths with psychopathic traits, higher levels of psychopathic traits were associated with cognitive perspective-taking deficits and, for females, there was no association with affective perspective-taking. It is worth noting that we cannot rule out that the failure to find relationships between perspective-taking and psychopathy may potentially be due to insufficient power to detect the small effect we found ${ }^{2}$.

A couple of factors should be kept in mind when considering our results. First, our sample consisted of younger individuals, with a maximum age of 13 . Whereas other researchers have thought of childhood psychopathy as extending downward to elementary school children $[6,11]$ or younger [8], assessing these particular outcomes in these younger, non-clinical-referred or non-'at-risk' samples had not previously been done, meaning there is very little research with which to compare our results.

2 A sensitivity analysis found that we could identify a small-moderate effect with a power of 0.80 given our sample size, but the effects found were much smaller and we would have needed a sample of nearly 300 to have power equal to 0.80 . 
More work is clearly needed with this population. With respect to the methodology, the significant relationships between components of the APSD and SDQ could arguably be partially due to the parent-report format of both measures. However, we believe that this does not account for the full relationships as the pattern of results are not spurious or weak (i.e., many of the relationships are moderate-to-high in magnitude, accounting for up to $40 \%$ of the variance, and remain even with stringent corrections made for multiple tests). The measures themselves are widely used and well-validated, arguably providing us with the best available assessments of both psychopathic traits and social-emotional well-being. We believe the validity and reliability of the two measures outweigh the potential drawbacks of using two parent-report measures.

In summary, through an examination of individual differences in psychopathic traits, the current study provides reasons for considering that the construct of psychopathy may change in meaning across development. Specifically, some correlates (i.e., between psychopathic traits and perspectivetaking) existing only at certain points whereas others (i.e., between psychopathic traits and social-emotional well-being) endure across ages and development. The current study failed to extend findings pertaining to perspective-taking deficits associated with psychopathy to a younger population, suggesting that the two are unrelated in community samples of elementary-school children or that this relationship may only emerge later in development. In contrast, individual differences in social-emotional functioning were found to be predictive of psychopathic traits using a multidimensional measure of socialemotional functioning (i.e., one that measured different aspects of social emotional functioning such as conduct problems, peer relationship problems, prosocial behavior, etc.). These results suggest that researchers and clinicians wishing to identify early warning signs of these traits might be best served by initially using multidimensional measures that assess a broad range of social skills rather than relying on a measure of any single aspect of social-emotional functioning.

\section{Acknowledgements}

The authors would like to thank first and foremost the participants and their families for their participation in this research. A special thank you to the schools that allowed us to come and conduct our research on site- we greatly appreciate it. We would also like to thank Sherilynn Chan, Winnie Chung, and Samantha Bangayan for their assistance with conducting this research. Funding for this research was provided in part by a fellowship to the first author by the Social Sciences and Humanities Research Council of Canada and by a University of British Columbia's Hampton Fund Research Grant to the second author.

\section{References}

1. Hare RD (1993) Without Conscience: the disturbing world of the psychopaths among us. The Guilford Press, New York.

2. Frick PJ, Bodin SD, Barry CT (2000) Psychopathic traits and conduct problems in community and clinic-referred samples of children: further development of the psychopathy screening device. Psychol Assess 12: 382-393.

3. Hare RD (1991) The hare psychopathy checklist-revised. Multi-Health Systems, Toronto

4. Poythress NG \& Hall JR (2011) Psychopathy and impulsivity reconsidered. Aggression and Violent Behavior 16: 120-134.

5. Cleckley H (1976) The mask of sanity. ( $5^{\text {th }}$ edn), St. Louis, Mosby, Missouri.

6. Barry CT, Frick PJ, DeShazo TM, McCoy MG, Ellis M, et al. (2000) The importance of callous-unemotional traits for extending the concept of psychopathy to children. J Abnorm Psychol 109: 335-340.

7. Harpur TJ, Hare RD (1994) Assessment of psychopathy as a function of age. J Abnorm Psychol 103: 604-609.

8. Bower B (2006) The predator's gaze: scientists explore the frightening world of psychopaths. Science News 170: 379-381.

9. Viding E, Blair RJ, Moffitt TE, Plomin R (2005) Evidence for substantial genetic risk for psychopathy in 7-year-olds. J Child Psychol Psychiatry 46: 592-597.

10. Block J (1993) Studying personality the long way. In D. C. Funder, R. D. Parke, Tomlinson-Keasey C, Widaman K (Eds.) Studying lives through time: personality and development. American Psychological Association, Washington DC, p. 9-41.

11. Fontaine NM, McCrory EJ, Boivin M, Moffitt TE, Viding E (2011) Predictors and outcomes of joint trajectories of callous-unemotional traits and conduct problems in childhood. J Abnorm Psychol 120: 730-742.

12. Frick PJ, Kimonis MS, Dandreaux DM, Farell JM (2003) The 4 year stability of psychopathic traits in non-referred youth. Behav Sci Law 21: 713-736.

13. Willoughby MT, Waschbusch DA, Moore GA, Propper CB (2011) Using the ASEBA to screen for callous unemotional traits in early childhood: factor structure, temporal stability, and utility. J Psychopathology Behav Assess 33: 19-30.

14. Frick PJ, Cornell AH, Barry CT, Bodin SD, Dane HE (2003) Callousunemotional traits and conduct problems in the prediction of conduct problem severity, aggression, and self-report of delinquency. J Abnorm Child Psychol 31: 457-470.

15.Loney BR, Taylor J, Butler MA, Iacono WG (2007) Adolescent psychopathy features: 6-year temporal stability and the prediction of externalizing symptoms during the transition to adulthood. Aggress Behav 33: 242-252.

16. Frick PJ, White SF (2008) Research review: the importance of callousunemotional traits for developmental models of aggressive and antisocial behavior. J Child Psychol Psychiatry 49: 359-375.

17. Fontaine NM, Rijsdijk FV, McCrory EJ, Viding E (2010) Etiology of different developmental trajectories of callous-unemotional traits. J Am Acad Child Adolesc Psychiatry 49: 656-664.

18. Blair RJ, Peschardt KS, Budhani S, Mitchell DG, Pine DS (2006) The development of psychopathy. J Child Psychol Psychiatry 47: 262-276.

19. American Psychiatric Association (2013) Diagnostic and statistical manual of mental disorders ( $5^{\text {th }}$ edn $)$, American Psychiatric Publishing, Arlington, VA.

20. Murrie DC, Marcus DK, Douglas KS, Lee Z, Salekin RT, et al. (2007) Youth with psychopathy features are not a discrete class: a taxometric analysis. J Child Psychol and Psychiatry 48: 714-723. 
21. Skilling TA, Quinsey VL, Craig WM (2001) Evidence of a taxon underlying serious antisocial behavior in boys. Criminal Justice and Behavior 28: 450-470.

22. Cohen D, Strayer J (1996) Empathy in conduct-disordered and comparison youth. Developmental Psychology 32: 988-998.

23. Loney BR, Frick PJ, Clements CB, Ellis ML, Kerlin K (2003) Callousunemotional traits, impulsivity, and emotional processing in adolescents with socially deviant behavior problems. Journal of Clinical Child and Adolescent Psychology 32: 66-80.

24. Blair RJR (2003) Neurobiological basis of psychopathy. The British Journal of Psychiatry 182: 5-7.

25. Blair RJ, Budhani S, Colledge E, Scott S (2005) Deafness to fear in boys with psychopathic tendencies. J Child Psychol Psychiatry 46: 327-336.

26. Blair RJ, Colledge E, Murray L, Mitchell DG (2001) A selective impairment in the processing of sad and fearful expressions in children with psychopathic tendencies. Journal of Abnormal Child Psychology 29: 491-498.

27. Mullins-Nelson JL, Salekin RT, Leistico AR (2006) Psychopathy, empathy, and perspective-taking in a community sample: implications for the successful psychopathy concept. International Journal of Forensic Mental Health 5: 133-149.

28. DeMatteo D, Heilbrun K, Marczyk G (2005) Psychopathy, risk of violence, and protective factors in a noninstitutionalized and noncriminal sample. International Journal of Forensic Mental Health 4: 147-157.

29. Blair RJR \& Coles M (2000) Expression recognition and behavioral problems in early adolescence. Cognitive Development 15: 421-434.

30.Woodcock RW, McGrew KS, Mather N (2001) Woodcock-Johnson III Brief Intellectual Assessment (WJ-III BIA). Riverside Publishing, Itasca.

31. Frick PJ, Hare RD (2001) The antisocial process screening device. Multi-Health Systems, Toronto.

32. Goodman R (1997) The strengths and difficulties questionnaire: a research note. J Child Psychol and Psychiatry 38: 581-586.

33. Bourdon KH, GoodmanR, Rae DS, SimpsonG, Koretz DS (2005) The strengths and difficulties questionnaire: U.S. normative data and psychometric properties. J Am Acad of Child Adolesc Psychiatry 44: 557-564.

34. Baron-Cohen S, Wheelwright S, Scahill V Lawson J, Spong A (2001) Reading the mind in the eyes - children's version. Cambridge University Press, Cambridge.

35. Cassels TG, Birch SAJ (under review) Comparisons of an open-ended vs. forced-choice 'mind reading' task: Implications for measuring perspective-taking and emotion recognition.

36. Carey, J Cassels TG (2013) Comparing two forms of a childhood perspective-taking measure using CFA and IRT. Psychol Assess 25: 879-892.

37. McGrew KS, Woodcock RW (2001) Technical manual: WoodcockJohnson III. Riverside, IL.

38. Barnett MA, Thompson S (1985) The role of perspective taking and empathy in children's Machiavellianism, prosocial behavior, and motive for helping. The Journal of Genetic Psychology 146: 295-305.

39. Eisenberg N, Fabes RA (1990) Empathy: Conceptualization, measurement, and relation to prosocial behavior. Motivation and Emotion 14: 131-149.

40. Kimonis ER, Frick PJ, Fazekas H, Loney BR (2006) Psychopathy, aggression, and the processing of emotional stimuli in non-referred girls and boys. Behav Sci Law 24: 21-37.

41. Babiak P, Neumann CS, Hare RD (2010) Corporate psychopathy: Talking the walk. Behavioral Sciences \& the Law 28: 174-193.

42. Dutton K (2o012) Wisdom from psychopaths. Scientific American Mind 23: 36-43.

43. Durand K, Gallay M, Seigneuric A, Robichon F, Baudouin JY (2007) The development of facial emotion recognition: the role of configural information. J Exp Child Psychol 97: 14-27.

44. Dadds MR, Hawes DJ, Frost AD, Vassallo S, Bunn P, et al. (2009) Learning to 'talk the talk': the relationship of psychopathic traits to deficits in empathy across childhood. J Child Psychol Psychiatry 50: 599-606. 